\title{
Lack of association between Toxoplasma gondii infection and hypertensive disorders in pregnancy: a case-control study in a Northern Mexican population
}

\author{
Cosme Alvarado-Esquivel ${ }^{1,6^{*}}$, Fernando Vázquez-Alaníz ${ }^{2}$, Ada A Sandoval-Carrillo ${ }^{3}$, José M Salas-Pacheco ${ }^{3}$, \\ Jesús Hernández-Tinoco ${ }^{1,3}$, Luis Francisco Sánchez-Anguiano ${ }^{1,3}$ and Oliver Liesenfeld ${ }^{4,5}$
}

\begin{abstract}
Background: The outcome of pregnancy is often threatened by hypertension disorders, i.e. eclampsia. Rate of infection with the protozoan parasite Toxoplasma gondii can be as high as 80\% in pregnant women, and infection acquired during pregnancy can lead to fetal death. Very little is known about a potential association between infections, i.e. those with Toxoplasma gondii, and hypertensive disorders during pregnancy.

Methods: Through a case-control study design, we investigated the presence of anti-Toxoplasma lgG and anti-Toxoplasma lgM antibodies in 146 pregnant women suffering from hypertensive disorders (cases) and 146 age-matched normotensive pregnant women (controls) attending a public hospital in Durango City, Mexico. Obstetric and blood pressure characteristics from cases and controls were also obtained.

Results: Seroprevalence of anti-Toxoplasma IgG antibodies and IgG titers did not differ significantly in controls $(8 / 146 ; 5.5 \%)$ and cases $(9 / 146 ; 6.2 \%)$. Anti-Toxoplasma lgM antibodies were found in $2(1.2 \%)$ controls and none of the cases. Seroprevalence of T. gondii in controls (5.5\%) was similar to seroprevalences found in patients with mild preeclampsia (4/27: 14.8\%), severe preeclampsia (5/95: 5.3\%), eclampsia (0/16: 0\%) and HELLP syndrome (hemolysis, elevated liver enzymes, and low platelet count) (0/8: $0 \%)(P=0.23)$.

Conclusions: Our results suggest that latent infection with $T$. gondii is not associated with hypertensive disorders in pregnant women in Northern Mexico. Further studies with larger sample sizes are needed to elucidate the association of infection with $T$. gondii with hypertensive disorders in pregnancy.
\end{abstract}

Keywords: Toxoplasma gondii, Seroprevalence, Preeclampsia, HELLP syndrome, Eclampsia, Infection, Epidemiology

\section{Background}

Hypertensive disorders in pregnancy cause maternal and perinatal morbidity and mortality $[1,2]$. About 8.5 million cases of preeclampsia are reported worldwide yearly [3]. The overall estimates of incidence in 40 countries including the 6 World Health Organization regions (African, European, Americas, Eastern Mediterranean, South-East Asia, and Western Pacific) were $4.6 \%$ and $1.4 \%$ of all

\footnotetext{
* Correspondence: alvaradocosme@yahoo.com

'Faculty of Medicine and Nutrition, Juárez University of Durango State,

Avenida Universidad S/N, Durango, Dgo 34000, Mexico

'Laboratorio de Investigación Biomédica, Facultad de Medicina y Nutrición,

Avenida Universidad S/N, Durango 34000, Dgo, Mexico

Full list of author information is available at the end of the article
}

deliveries for preeclampsia and eclampsia, respectively [4]. In Mexico, eclampsia and preeclampsia are recognized as direct causes of maternal deaths [5]. The etiology and primary pathology of preeclampsia remain elusive [1]. The role of infection in preeclampsia remains controversial. A body of evidence supports a link between preeclampsia and maternal infection [6,7]. For instance, in a systematic review of epidemiological studies, researchers found that any bacterial or viral infection was associated with a twofold higher risk of developing preeclampsia compared to women without infection [6]. Furthermore, in a metaanalysis of 49 studies, urinary tract infection and periodontal disease during pregnancy were associated with

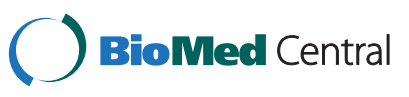


an increased risk of preeclampsia [7]. More recent studies have recognized infections with high risk human papilloma virus [8], Chlamydia trachomatis [9], periodontitis [10], as well as Chlamydia pneumoniae [11] and cytomegalovirus IgG seropositivity [12] as risk factors for preeclampsia. On the other hand, some studies have not found an association of preeclampsia with infection with cytomegalovirus [13,14], Chlamydia pneumonia, Herpes simplex virus 2 [14], or respiratory tract infection [15]. HBsAg carriage [16] has even been found to be associated with a reduced incidence of preeclampsia.

Primary infection with $T$. gondii during pregnancy represents a risk for congenital disease $[17,18]$. The association of infection with $T$. gondii and preeclampsia has been insufficiently studied. Therefore, we performed a case-control study in Northern Mexico to determine the association between $T$. gondii infection and preeclampsia among pregnant women attending the Department of Gynecology and Obstetrics in a secondary-care public hospital in Durango City, Mexico.

\section{Methods}

Study design and study populations

Through a case-control study design, we studied the association of $T$. gondii infection with hypertensive disorders in pregnant women in Durango City, Mexico from November 2011 to September 2013. We used a 1:1 ratio for matching. Cases and controls were matched by age, gender, attending hospital, and residence.

\section{Women with hypertensive disorders in pregnancy}

Inclusion criteria for cases were: 1) women with 2242 weeks of pregnancy suffering from hypertensive disorders and proteinuria attended in the Department of Gynecology and Obstetrics of a public secondary-care hospital (General Hospital) in Durango City, Mexico; and 2) who agreed to participate in the study. Hypertensive disorders included: 1) mild preeclampsia (blood pressure $\geq 140 / 90 \mathrm{mmHg}$ on 2 occasions, at least 6 hours apart, and proteinuria of $\geq 300 \mathrm{mg} / 24$ hours); 2) severe preeclampsia (blood pressure $\geq 160 / 110 \mathrm{mmHg}$ on 2 occasions, at least 6 hours apart, and proteinuria of $\geq 5 \mathrm{~g} / 24$ hours); 3 ) eclampsia (hypertension, proteinuria and seizures); and 4) HELLP syndrome (hypertension, proteinuria and presence of hemolytic anemia, elevated liver enzymes and low platelet count). As a sampling strategy, all eligible women attended during the study period were invited to participate. In total, 146 cases accepted to participate in the study. All patients resided in Durango City.

\section{Control subjects}

Inclusion criteria for controls were: 1) pregnant women without hypertensive disorders, nephropathy, or diabetes before or early during pregnancy attended in the same
Department of Gynecology and Obstetrics of The General Hospital in Durango City as cases; 2) normal pregnancy with normal blood pressure levels (systolic blood pressure $<140 \mathrm{mmHg}$ and diastolic blood pressure <90 mmHg); 3) without any underlying disease; and 4) who agreed to participate in the study. In total, 146 controls were included in the study. Controls were selected days after cases were included in the study.

Clinical data obtained from the patients (cases and controls) included age, number of pregnancies, deliveries, cesarean sections, miscarriages and stillbirths, week of pregnancy, history of preeclampsia, systolic and diastolic blood pressures, and mean arterial pressure.

\section{Ethical aspects}

This study was approved by the Institutional Ethical Committee of The General Hospital of the Secretary of Health in Durango City, Mexico. The purpose and procedures of the study were explained to all participants, and a written informed consent was obtained from all of them.

\section{Laboratory tests}

A blood sample of about $7 \mathrm{~mL}$ was obtained by venipuncture from the median cubital vein of each participant. Blood was centrifuged and serum samples were obtained and kept frozen at $-20^{\circ} \mathrm{C}$ until analyzed. Sera were analyzed by qualitative and quantitative methods for anti-T. gondii IgG antibodies with the commercially available enzyme immunoassay kit "Toxoplasma IgG" (Diagnostic Automation Inc., Calabasas, CA, USA). AntiT. gondii IgG antibody levels were expressed as International Units $(\mathrm{IU}) / \mathrm{ml}$, and a result equal to or greater than $8 \mathrm{IU} / \mathrm{ml}$ was considered positive. Sera positive for anti-T. gondii IgG antibodies were further analyzed for anti-T. gondii IgM antibodies by the commercially available enzyme immunoassay "Toxoplasma IgM" kit (Diagnostic Automation Inc., Calabasas, CA, USA). The cut off for anti- $T$. gondii IgM antibody positivity was obtained by multiplying the absorbance of the calibrator by the factor (0.40) recommended in the kit. We have used similar cut-offs for positivity to IgG and IgM antibodies in epidemiological studies in the region $[19,20]$. All tests were performed following the instructions of the manufacturer.

\section{Statistical analysis}

Results were analyzed with the aid of Epi Info versions 3.5.4 and 7, and SPSS 15.0 (SPSS Inc. Chicago, Illinois). For calculation of the sample size, we used a 95\% confidence level, a power of $80 \%$, a $1: 1$ proportion of cases and controls, a reference seroprevalence of $6.1 \%$ [21] as the expected frequency of exposure in controls, and an odds ratio of 3.3. The result of the sample size calculation was 139 cases and 139 controls. A number of 
appropriate statistical methods for the study design [22] were used. Age values among the groups were compared by the paired student's $t$ test. For comparison of the frequencies of seropositivity to T. gondii and obstetric characteristics between cases and controls, the McNemar's test was used. Odds ratio (OR) and 95\% confidence interval (CI) were calculated by the Mantel-Haenszel analysis. We used the Pearson's chi-squared test and the two-tailed Fisher's exact test (when values were less than 5) to assess the association of T. gondii seropositivity and clinical and obstetric characteristics of the women studied. Statistical significance was set at a $P$ value less than 0.05 .

\section{Results}

Of the 146 cases included in the study, 27 suffered from mild preeclampsia, 95 from severe preeclampsia, 16 from eclampsia, and 8 from HELLP syndrome. The mean age of the cases was $23.51 \pm 6.41$ years (range: $15-$ 39 years) and the mean age of the controls was $23.44 \pm$ 6.17 (range: 15-39). There was no statistically significant difference in age between cases and controls $(P=0.92)$.

Anti-T. gondii IgG antibodies were found in 8 (5.5\%) of 146 controls and in 9 (6.2\%) of 146 cases. There was not difference in $T$. gondii infection among case control pairs (Table 1). Of the 8 anti-T. gondii IgG positive control patients, $5(3.4 \%)$ had IgG levels higher than $150 \mathrm{IU} / \mathrm{ml}$, and $3(2.1 \%)$ between 8 to $99 \mathrm{IU} / \mathrm{ml}$. In comparison, of the 9 anti-T. gondii IgG positive cases, 6 (4.1\%) had IgG levels higher than $150 \mathrm{IU} / \mathrm{ml}$, and 3 (2.1\%) between 8 to $99 \mathrm{IU} / \mathrm{ml}$. Anti-T. gondii IgG levels in controls were similar to those in cases $(\mathrm{OR}=1.20$; 95\% CI: 0.36-3.93; $P=0.76)$. Anti-T. gondii IgM antibodies were found in $2(1.4 \%)$ controls but none were found in the cases in this study.

With respect to clinical characteristics, seroprevalence of T. gondii in controls (5.5\%) was similar to the seroprevalence found in cases with mild preeclampsia (4/27: 14.8\%), severe preeclampsia (5/95: $5.3 \%)$, eclampsia (0/16: $0 \%)$ and HELLP syndrome $(0 / 8: 0 \%)(P=0.23)$. Of the obstetric characteristics, seropositivity to $T$. gondii was not associated with the deliveries, cesarean sections, miscarriages or a history of preeclampsia. In contrast, seroprevalence of $T$. gondii infection was significantly $(P=0.01)$ higher in women with more than one pregnancy (14/150: 9.3\%) than in women with only one pregnancy (3/142: $2.1 \%)$. In addition, women with a history of stillbirths had a significantly $(P=0.009)$ higher $(2 / 3: 66.7 \%)$ seroprevalence of $T$. gondii infection than women without such history (15/289: 5.2\%). On the other hand, comparison of obstetric characteristics in cases and controls pairs showed that preeclampsia was positively associated with a history of preeclampsia and cesarean sections and negatively associated with having more than one pregnancy and a history of deliveries (Table 2).

\section{Discussion}

At present, very little is known about the role of infections in hypertension disease during pregnancy. This study was performed to investigate a potential association of infection with the protozoan parasite $T$. gondii with hypertensive disorders in pregnancy. Infections with T. gondii cause an increase in dopamine [23,24], and it is known that dopamine increases the blood pressure [25]. However, none of the serological markers of $T$. gondii infection, i.e., presence of anti-T. gondii IgG and IgM antibodies, and levels of anti-T. gondii IgG antibodies used in the present study correlated with hypertensive disorders in the pregnant women studied. In addition, seropositivity to $T$. gondii was also observed in similar frequencies between women with a history of preeclampsia and women without such history. Therefore, our results indicate that seropositivity to $T$. gondii is not likely to substantially contribute in the etiology of hypertensive disorders in pregnancy in our population.

Only two studies have investigated a potential association between infection with $T$. gondii and hypertension disorders, none of which observed an apparent association $[11,26]$. In a serological study in pregnant women in Norway, researchers found that women seronegative for Herpes simplex virus type 2, cytomegalovirus, and Epstein Barr virus had an increased risk for developing preeclampsia while seronegativity to $T$. gondii did not [26]. In a study in Turkey, seroprevalence of anti-T. gondii antibodies were similar in 54 preeclampsia women and 54 healthy pregnant women [11]. However, such studies only reported qualitative results on the seropositivity rate of infection in preeclampsia patients. In the present study, we performed a quantitative determination of anti- $T$. gondii IgG antibodies. Our results suggest that antibody concentrations were not related with hypertension in pregnant women. We studied mostly patients suffering from severe preeclampsia and

Table 1 Distribution of T. gondii infection among case control pairs

\begin{tabular}{lccccc}
\hline Case & \multicolumn{2}{c}{ Control } & Total & OR [95\% CI] & \multirow{p}{*}{-value } \\
\cline { 2 - 4 } & Exposed (T. gondii infection) & Unexposed (No T. gondii infection) & & \\
\hline Exposed (T. gondii infection) & 8 & 1 & 137 & \\
Unexposed (No T. gondii infection) & 0 & 138 & 137 & \\
Total & 8 & 146 & $3.0[0.12-73.64]$ & 1 \\
\hline
\end{tabular}


Table 2 Distribution of obstetric characteristics among case control pairs

\begin{tabular}{|c|c|c|c|c|c|c|}
\hline \multirow{2}{*}{$\begin{array}{l}\text { Characteristic } \\
\text { Pregnancy history }\end{array}$} & \multirow[t]{2}{*}{ Case } & \multicolumn{2}{|c|}{ Control } & \multirow[t]{2}{*}{ Total } & \multirow[t]{2}{*}{ OR $[95 \% \mathrm{Cl}]$} & \multirow[t]{2}{*}{$p$-value } \\
\hline & & Exposed (More than 1 pregnancy) & Unexposed (One pregnancy) & & & \\
\hline & Exposed (More than 1 pregnancy) & 71 & 0 & 71 & & \\
\hline & Unexposed (One pregnancy) & 8 & 67 & 75 & & \\
\hline & Total & 79 & 67 & 146 & $0.05[0.00-0.70]$ & 0.01 \\
\hline \multirow[t]{4}{*}{ History of deliveries } & & Exposed (History of delivery) & Unexposed (No history of delivery) & & & \\
\hline & Exposed (History of delivery) & 65 & 0 & 65 & & \\
\hline & Unexposed (No history of delivery) & 13 & 68 & 81 & & \\
\hline & Total & 78 & 68 & 146 & $0.03[0.00-0.40]$ & 0.001 \\
\hline \multirow[t]{4}{*}{ Cesarean sections } & & Exposed (Cesarean section) & Unexposed (No Cesarean section) & & & \\
\hline & Exposed (Cesarean section) & 36 & 66 & 102 & & \\
\hline & Unexposed (No Cesarean section) & 0 & 44 & 44 & & \\
\hline & Total & 36 & 110 & 146 & $133.0[8.23-2148.67]$ & $<0.0001$ \\
\hline \multirow[t]{4}{*}{ History of miscarriage } & & Exposed (Miscarriage) & Unexposed (No miscarriage) & & & \\
\hline & Exposed (Miscarriage) & 21 & 4 & 25 & & \\
\hline & Unexposed (No miscarriage) & 0 & 121 & 121 & & \\
\hline & Total & 21 & 125 & 146 & $9.0[0.48-167.17]$ & 0.17 \\
\hline \multirow[t]{4}{*}{ History of stillbirth } & & Exposed (Stillbirth) & Unexposed (No stillbirth) & & & \\
\hline & Exposed (Stillbirth) & 0 & 3 & 3 & & \\
\hline & Unexposed (No stillbirth) & 0 & 143 & 143 & & \\
\hline & Total & 0 & 146 & 146 & $7.0[0.36-135.52]$ & 0.31 \\
\hline \multirow[t]{4}{*}{ History of preeclampsia } & & Exposed (Preeclampsia) & Unexposed (No preeclampsia) & & & \\
\hline & Exposed (Preeclampsia) & 6 & 15 & 21 & & \\
\hline & Unexposed (No preeclampsia) & 0 & 124 & 124 & & \\
\hline & Total & 6 & 139 & 145 & $31.0[1.85-518.11]$ & 0.0004 \\
\hline
\end{tabular}


small subgroups of hypertension disease including mild preeclampsia, eclampsia and HELLP syndrome. Therefore, the association of infection with $T$. gondii with mild preeclampsia, eclampsia and HELLP syndrome should be further investigated with larger sample sizes. There are still some questions to answer about the role of $T$. gondii infection in hypertension disease in pregnancy. In a case control study, treatment of pregnant women with spiramycin, a macrolide antibiotic administered to women with suspected infection before 18 weeks of pregnancy to reduce the rate of transmission of the parasite to the fetus [17] - reduced the incidence of pregnancy-induced hypertension [27]. In this study, researchers studied a cohort of 417 pregnant women treated with spiramycin because of seroconversion for $T$. gondii and 353 low-risk women who did not take any antibiotic during pregnancy, and observed that treated women had a lower risk of developing pregnancy-induced hypertension than untreated women. It is not clear whether such treatment directly impacted on the blood pressure; the authors hypothesized that the spiramycin treatment lowers the risk of developing preeclampsia probably by preventing the onset of infections that could complicate pregnancy [27]. Studies about the effects of dopamine on blood pressure in T. gondii infected subjects should be conducted.

\section{Conclusions}

Results of the present study indicate that seropositivity to $T$. gondii was not associated with hypertensive disorders in pregnant women attending an obstetrics and gynecology department in Northern Mexico. However, due to the small sample size further studies with larger sample sizes are needed to elucidate the association of T. gondii infection with hypertension disease during pregnancy.

\section{Competing interests}

The authors declare that they have no competing interests. Oliver Liesenfeld is Chief Medical Officer at Roche Molecular Systems.

\section{Authors' contributions}

CAE conceived and designed the study protocol, performed the laboratory tests and data analysis, and wrote the manuscript. FVA, AASC, and JMSP obtained the blood samples and clinical data, and performed the data analysis. JHT, LFSA and OL performed the data analysis and wrote the manuscript. All authors read and approved the final version of the manuscript.

\section{Acknowledgment}

This study was financially supported by Juarez University of Durango State.

\section{Author details}

${ }^{1}$ Faculty of Medicine and Nutrition, Juárez University of Durango State, Avenida Universidad S/N, Durango, Dgo 34000, Mexico. ${ }^{2}$ Hospital General de Durango, Secretaría de Salud, Avenida 5 de febrero y Norman Fuentes, Durango, Dgo 34000, Mexico. ${ }^{3}$ Institute for Scientific Research "Dr. Roberto Rivera Damm", Juárez University of Durango State, Avenida Universidad S/N, Durango, Durango 34000, Mexico. ${ }^{4}$ Institute for Microbiology and Hygiene, Campus Benjamin Franklin, Charité Medical School, Hindenburgdamm 27, Berlin D-12203, Germany. ${ }^{5}$ Present address: Roche Molecular Diagnostics,
Pleasanton, CA, USA. 'aboratorio de Investigación Biomédica, Facultad de Medicina y Nutrición, Avenida Universidad S/N, Durango 34000, Dgo, Mexico.

Received: 13 January 2014 Accepted: 30 March 2014

Published: 3 April 2014

\section{References}

1. Jido TA, Yakasai IA: Preeclampsia: a review of the evidence. Ann Afr Med 2013, 12:75-85. doi: 10.4103/1596-3519.112395.

2. Lo JO, Mission JF, Caughey AB: Hypertensive disease of pregnancy and maternal mortality. Curr Opin Obstet Gynecol 2013, 25:124-132. doi:10.1097/GCO.0b013e32835e0ef5

3. Anderson UD, Olsson MG, Kristensen KH, Åkerström B, Hansson SR: Review: Biochemical markers to predict preeclampsia. Placenta 2012, 33(Suppl):S42-S47. doi:10.1016/j.placenta.2011.11.021.

4. Abalos E, Cuesta C, Grosso AL, Chou D, Say L: Global and regional estimates of preeclampsia and eclampsia: a systematic review. Eur J Obstet Gynecol Reprod Biol 2013, 170:1-7. doi:10.1016/j.ejogrb.2013.05.005.

5. Fajardo-Dolci G, Meljem-Moctezuma J, Vicente-González E, Venegas-Páez FV, Villalba-Espinoza I, Pérez-Cardoso AL, Barrón-Saldaña DA, Montesinos-Gómez G, Aguirre Güemez AV, Novoa-Boldo A, Mendoza-Larios LA, López-González R, Mazón-González B, Aguirre-Gas HG: Analysis of maternal deaths in Mexico occurred during 2009. Rev Med Inst Mex Seguro Soc 2013, 51:486-495.

6. Rustveld LO, Kelsey SF, Sharma R: Association between maternal infections and preeclampsia: a systematic review of epidemiologic studies. Matern Child Health J 2008, 12:223-242.

7. Conde-Agudelo A, Villar J, Lindheimer M: Maternal infection and risk of preeclampsia: systematic review and metaanalysis. Am J Obstet Gynecol 2008, 198:7-22. doi:10.1016/j.ajog.2007.07.040.

8. McDonnold M, Dunn H, Hester A, Pacheco LD, Hankins GD, Saade GR, Costantine MM: High risk human papillomavirus at entry to prenatal care and risk of preeclampsia. Am J Obstet Gynecol 2014, 210:138.e1-5. doi:10.1016/j.ajog.2013.09.040.

9. Haggerty CL, Klebanoff MA, Panum I, Uldum SA, Bass DC, Olsen J, Roberts JM, Ness RB: Prenatal Chlamydia trachomatis infection increases the risk of preeclampsia. Pregnancy Hypertens 2013, 3:151-154.

10. Moura da Silva G, Coutinho SB, Piscoya MD, Ximenes RA, Jamelli SR: Periodontitis as a risk factor for preeclampsia. J Periodontol 2012, 83:1388-1396. doi:10.1902/jop.2012.110256.

11. Soydinc HE, Kan I, Dal T, Evsen MS, Sak ME, Ozler A, Turgut A, Yildiz I: Evaluation of the relationship between preeclampsia and seropositivity of infectious disease in maternal plasma. Clin Ter 2013, 164:e199-e202. doi:10.7417/CT.2013.1568

12. Xie F, Hu Y, Magee LA, Money DM, Patrick DM, Krajden M, Thomas E, von Dadelszen P, Toxemia Study Group: An association between cytomegalovirus infection and pre-eclampsia: a case-control study and data synthesis. Acta Obstet Gynecol Scand 2010, 89:1162-1167. doi:10.3109/00016349.2010.499449.

13. Strand KM, Odland ML, Iversen AC, Nordbø SA, Vik T, Austgulen R: Cytomegalovirus antibody status at 17-18 weeks of gestation and pre-eclampsia: a case-control study of pregnant women in Norway. BJOG 2012, 119:1316-1323. doi:10.1111/j.1471-0528.2012.03420.x.

14. Zhang JF, Zhang WY: Relationship of cytomegalovirus, Chlamydia pneumoniae and herpes simplex virus type 2 infections with preeclampsia. Zhonghua Yi Xue Za Zhi 2012, 92:1413-1415.

15. Minassian C, Thomas SL, Williams DJ, Campbell O, Smeeth L: Acute maternal infection and risk of pre-eclampsia: a population-based casecontrol study. PLoS One 2013, 8:e73047. doi:10.1371/journal.pone.0073047.

16. Lao TT, Sahota DS, Cheng YK, Law LW, Leung TY: Maternal hepatitis B surface antigen status and incidence of pre-eclampsia. J Viral Hepat 2013, 20:343-349. doi:10.1111/jvh.12037.

17. Montoya JG, Liesenfeld O: Toxoplasmosis. Lancet 2004, 363:1965-1976.

18. Mwambe B, Mshana SE, Kidenya BR, Massinde AN, Mazigo HD, Michael D, Majinge C, Groß U: Sero-prevalence and factors associated with Toxoplasma gondii infection among pregnant women attending antenatal care in Mwanza, Tanzania. Parasit Vectors 2013, 6:222. doi:10.1186/1756-3305-6-222.

19. Alvarado-Esquivel C, Torres-Castorena A, Liesenfeld O, Estrada-Martínez S, Urbina-Álvarez JD: High seroprevalence of Toxoplasma gondii infection in 
a subset of Mexican patients with work accidents and low socioeconomic status. Parasit Vectors 2012, 5:13. doi:10.1186/1756-3305-5-13.

20. Alvarado-Esquivel C, Campillo-Ruiz F, Liesenfeld O: Seroepidemiology of infection with Toxoplasma gondii in migrant agricultural workers living in poverty in Durango, Mexico. Parasit Vectors 2013, 6:113.

21. Alvarado-Esquivel C, Sifuentes-Alvarez A, Narro-Duarte SG, Estrada-Martínez S, Díaz-García JH, Liesenfeld O, Martínez-García SA, Canales-Molina A: Seroepidemiology of Toxoplasma gondii infection in pregnant women in a public hospital in northern Mexico. BMC Infect Dis 2006, 6:113.

22. Niven DJ, Berthiaume LR, Fick GH, Laupland KB: Matched case-control studies: a review of reported statistical methodology. Clin Epidemiol 2012, 4:99-110. doi:10.2147/CLEP.S30816.

23. Prandovszky E, Gaskell E, Martin H, Dubey JP, Webster JP, McConkey GA: The neurotropic parasite Toxoplasma gondii increases dopamine metabolism. PLoS One 2011, 6:e23866. doi:10.1371/journal.pone.0023866.

24. Flegr J: How and why Toxoplasma makes us crazy. Trends Parasitol 2013, 29:156-163. doi:10.1016/j.pt.2013.01.007.

25. Tayebati SK, Lokhandwala MF, Amenta F: Dopamine and vascular dynamics control: present status and future perspectives. Curr Neurovasc Res 2011, 8:246-257.

26. Trogstad LI, Eskild A, Bruu AL, Jeansson S, Jenum PA: Is preeclampsia an infectious disease? Acta Obstet Gynecol Scand 2001, 80:1036-1038.

27. Todros T, Verdiglione $P$, Oggè G, Paladini D, Vergani P, Cardaropoli S Low incidence of hypertensive disorders of pregnancy in women treated with spiramycin for Toxoplasma infection. Br J Clin Pharmacol 2006, 61:336-340.

doi:10.1186/1756-3305-7-167

Cite this article as: Alvarado-Esquivel et al:: Lack of association between Toxoplasma gondii infection and hypertensive disorders in pregnancy: a case-control study in a Northern Mexican population. Parasites \& Vectors 2014 7:167.

\section{Submit your next manuscript to BioMed Central and take full advantage of:}

- Convenient online submission

- Thorough peer review

- No space constraints or color figure charges

- Immediate publication on acceptance

- Inclusion in PubMed, CAS, Scopus and Google Scholar

- Research which is freely available for redistribution 